Arroyo González, R. y Jiménez-Baena, A. F. (2016). Estruturación del ensayo científico sobre contenidos interculturāes y competencias escritoras en estudiantes universitarios. Revista de Investigación Educativa, 34(2), 351-367. DOI: http://dx.doi.org/10.6018/rie.34.2.230611

\title{
Estructuración del ensayo científico sobre contenidos interculturales y competencias escritoras en estudiantes universitarios
}

\author{
The structuration of the scientific essay about intercultural \\ contents and writing competences with university students
}

\author{
Rosario Arroyo González y Abrahan Fco. Jiménez-Baena \\ Departamento de Didáctica y Organización Escolar, Facultad de Ciencias de la Educación. \\ Universidad de Granada. España
}

\begin{abstract}
Resumen
Organizar un texto de forma argumentativa, es una demanda de primer orden en las tareas académicas universitarias, porque: a) ayuda al estudiante a sintetizar ideas y a profundizar en la compresión y expresión de sus propios conocimientos, en cualquier área; y b) contribuye, de un modo decisivo, a la construcción de su identidad cultural desde una perspectiva crítica. En esta investigación se aplica una metodología multimétodo, concretamente: a) el análisis de contenido, usando el programa Nvivo, para describir las estructuras gramaticales, los contenidos interculturales y las competencias escritoras, expresadas por una muestra de estudiantes; y $b$ ) el análisis estadístico con el programa SPSS para descubrir la correlación entre las competencias escritoras y los elementos estructurales. Se concluye con las necesidades de formación de los estudiantes en niveles universitarios para el desarrollo de la composición argumentativa, y una propuesta didáctica que potencie las competencias comunicativas escritas interculturales.

Palabras clave: competencias escritoras; ensayo científico; estudiantes universitarios; contenidos interculturales.
\end{abstract}

Correspondencia: Dra. Rosario Arroyo González, Campus Universitario de Cartuja s/n 18190 Granada. E-mail: rarroyo@ugr.es ; D. Abrahan Jiménez Baena, Campus Universitario de Cartuja s/n 18190 Granada. E-mail: brancus@correo.ugr.es 


\begin{abstract}
Organizing a text, in an argumentative form, is a first-order demand in university tasks because: a) it helps students to synthesize ideas and deepen the understanding and expression of their own knowledge in any area, and $b$ ) it contributes decisively to the construction of the cultural identity, from a critical perspective. A multimethod methodology is applied in this research, specifically: a) content analysis using NVivo software to describe grammatical structures and writing competences; and b) statistical analysis using SPSS program to find the correlation between writing competences and the expressed structural elements. It concludes with the training needs of students for the development of the argumentative composition, and a didactic proposal that fosters intercultural written communicative competences.

Keywords: writing competences; scientific essay; university students; intercultural contents.
\end{abstract}

\title{
Introducción
}

Because the writing process is complex, requiring the coordination of multiple and simultaneously occurring cognitive elements, such apprenticeship models are forms of instruction that allow teachers to scaffolds discrete thinking processes for students, and give students progressively more responsibility for decision-making over a series of lessons. (De la Paz \& McCutchen, 2010, p. 33).

Como se señala, la escritura es una tarea compleja que exige un progresivo proceso de enseñanza en todos los niveles académicos. Más concretamente, la demanda de múltiples documentos escritos en la universidad está contribuyendo a un cambio de percepción en la enseñanza en este nivel (Dysthe, 2007). Esto ha conducido a entender que la escritura universitaria es mucho más que el dominio de unas habilidades comunicativas. Se trata, en realidad, de una competencia indispensable para la reflexión, la adquisición y la recreación del conocimiento científico y profesional.

\section{Antecedentes}

La escritura en el nivel educativo universitario debe reunir criterios de calidad. Estos criterios "requires command of relatively static and conventionalised linguistic and structural structures... to achieve one or more of a variety of purposes...within a socio-cultural context" (Gavin \& Marshall, 2012, p. 653). Se refieren, estos autores, al dominio de convencionalismos lingüísticos y estructuras textuales para lograr unos propósitos concretos dentro de un contexto socio-cultural determinado. En este sentido desde el contexto laboral se destaca que los profesionales hábiles en competencias escritas, lideran las interacciones interpersonales y las prácticas empresariales (Cleaveland \& Larkins, 2004), por lo que, las competencias comunicativas escrita de alto nivel se configura como una de las dimensiones de desarrollo más decisivas en la promoción profesional e investigadora.

Con respecto a las competencias escritoras que deberían conocer y dominar los estudiantes universitarios, para el ejercicio de una profesión; un estudio de la Universidad de Granada (Arroyo, 2013) destaca que las exigencias de la escritura de calidad, 
reclaman un profundo esfuerzo cognitivo y un gran interés emocional que dinamice la voluntad y autorregule este aprendizaje. En consecuencia, cuando los escritores universitarios producen un texto, no sólo deben de conocer los patrones gramaticales del mismo, sino que, sobre todo, deben prestar atención a cómo, ese texto está mediatizado por el contexto en el que se desarrolla; ajustándose a los diferentes requerimientos académicos y a las demandas de los usos tecnológicos y profesionales de la escritura. $\mathrm{Y}$ todo esto ocurre sea cual fuere el idioma que se utilice.

Basándose en el "Modelo Metasociocognitivo de la Composición Escrita" (Arroyo, 2009, p.61), que comprende la composición de textos como interacción de procesos de diferente naturaleza (Salvador, 2005; Bazerman, 2008; Myhill \& Fisher, 2010);

"we claim that writing expertise may be conceptualized as having two dimensions: (1) the kinds of knowledge writers need to draw from when working on a specific text, and (2) the competencies writers need to perform when putting their knowledge into action" (Beaufort \& Iñesta, 2014, p.146).

En definitiva se identifica la escritura, en cualquier lengua, como un complejo proceso lingüístico, metacognitivo y social, en el que se integran diversas competencias de forma recursiva:

Estas son:

1. Competencias de planificación, de transcripción y de revisión de la escritura.

2. Competencias de autorregulación, de elaboración de una teoría de la tarea escritora y de una teoría del texto.

3. Competencias sociopolíticas, comunitarias-profesionales y de construcción de la identidad en la comunicación escrita.

4. Competencias de autocontrol emocional, de motivación y de creatividad en la expresión escrita.

Por lo tanto, se destaca que el estudiante universitario ha de conocer estas competencias escritoras y ponerlas en práctica, para producir escritos de calidad y, en concreto, para construir textos argumentativos (Torrace, Thomas \& Robinson, 2000; Kieft, Rijlaarsdam \& Van Den Bergh, 2006). Esto es así, porque organizar un texto de forma argumentativa, es una demanda de primer orden en las tareas académicas $\mathrm{y}$, además, ayuda al individuo a sintetizar ideas y a profundizar en la compresión y expresión de sus propios conocimiento. Diversos autores señalan que un componente esencial del texto argumentativo es tener claros los objetivos y formular argumentos, contraargumentos y refutaciones (Nussbaum \& Kardash, 2005).

Así pues, en perspectiva normativa los elementos esenciales del ensayo científico o texto argumentativo en el nivel académico universitario, deben ser: a) introducción al problema; b) premisa; c) razones para apoyar la premisa; d) posiciones en contra; e) razones para refutar los contraargumentos; f) ejemplo; g) conclusión justificada (Salvador, 2008). Además, los estudios de Takao \& Kelly (2003) y de Venables \& Summit (2003), ponen de manifiesto la necesidad de usar investigaciones, citas de expertos; y evidencias en los ensayos científicos. 


\section{Finalidad}

Coherente con los antecedentes de investigación presentados, este artículo describe la estructura y contenidos interculturales de los ensayos científicos escritos por estudiantes universitarios. Además, se comprueba en qué medida correlaciona la organización estructural del ensayo científico con el conocimiento que el estudiante posee sobre competencias escritoras, todo ello, con el fin de identificar necesidades formativas que fundamenten el diseño y aplicación de programas para la enseñanza del ensayo científico en el nivel académico universitario.

Sin duda, el reto de esta línea de investigación, es lograr que la enseñanza universitaria de respuesta a las nuevas necesidades de las sociedades tecnologizadas del Siglo XXI y en las que se exige la formación de los ciudadanos en competencia multilingüe y tecnológica (Cernadas, Santos \& Lorenzo, 2013).

\section{Método}

Esta investigación utiliza instrumentos de recogida de datos cualitativos, sin embargo, aplica instrumentos de análisis tanto cualitativo como cuantitativo para un conocimiento más profundo de los casos analizados (Tashakkori \& Teddlie, 2003), tal y como se recoge en la Tabla 1.

Tabla 1

Diseño de investigación mixto

\begin{tabular}{lll}
\hline Instrumentos & Datos & Análisis \\
\hline $\begin{array}{l}\text { Plantillas con los ensayos escritos } \\
\text { por los estudiantes }\end{array}$ & Datos textuales & Sistema de Categorías (Programa \\
Nvivo) \\
$\begin{array}{l}\text { Entrevista sobre competencias es- } \\
\text { critoras }\end{array}$ & Frecuencias & $\begin{array}{l}\text { Matrices de frecuencias (Programa } \\
\end{array}$ \\
& & Evivo) \\
& & Estadísticos (Programa SPSS) \\
\hline
\end{tabular}

\section{Objetivos e hipótesis}

Para lograr el fin propuesto en esta investigación, se especifican los siguientes objetivos:

1. Analizar elementos estructurales expresados en los ensayos científicos producidos por estudiantes universitarios de diferentes niveles, así como el contenido expresado en los mismos.

2. Identificar las competencias escritoras que el estudiante universitario, de diferentes niveles, expresa mediante la evocación del pensamiento declarativo sobre operaciones escritoras. 
3. Descubrir la correlación entre los elementos estructurales de los ensayo científico y el conocimiento de competencias escritoras expresado por el estudiante universitario

4. Detectar necesidades formativas del estudiante universitario en la construcción de ensayos científicos

Las hipótesis que han guiado este estudio han sido

H1. El conocimiento de competencias escritoras expresado por una muestra de estudiantes universitarios no correlaciona con los elementos estructurales de los ensayos científicos producidos por ellos mismos.

H2. Los elementos estructurales de los ensayos científicos escritos por estudiantes universitarios de cursos diferentes no presentan diferencias significativas.

Para alcanzar estos objetivos y comprobar estas hipótesis se plantea un diseño de investigación mixto o multimétodo.

\section{Población y muestra}

En la selección de la muestra (véase Tabla 2) se aplicó un muestreo no probabilístico deliberado y opinático (Mayorga \& Ruiz Baeza, 2002).

Tabla 2

Participantes de la investigación

\begin{tabular}{lll}
\hline Nivel Académico & Cantidad & Características \\
\hline 67\% primer curso & 43 & $74 \%$ mujeres / 26\% hombres \\
de Universidad & & $35 \% 18$ años \\
$32 \%$ tercer y cuarto curso & $45 \% 19$ años \\
de Universidad & $20 \%$ entre 23 y 27 años \\
\hline
\end{tabular}

Se eligió este tipo de muestreo en base a los objetivos de la investigación, que pretenden realizar un estudio en profundidad de los productos escritos científicos de estudiantes universitarios y cómo estos correlacionan con su conocimiento en competencias escritoras.

Seguidamente se describirá el procedimiento de selección de la muestra, así como, los procedimientos de recogida, análisis y reducción de los datos.

\section{Instrumentos}

La técnica aplicada para la recogida de datos fue, por un lado, los ensayos escritos de los estudiantes $\mathrm{y}$, por otro, la encuesta, en su modalidad de entrevista cognitiva estructurada escrita. 
El procedimiento aplicado para la elaboración de esta entrevista fue el grupo de discusión en base a entrevistas cognitivas previas elaboradas por Graham \& Harris, (2005) y Salvador (2005). Los 37 ítems, que componen esta entrevista, se pensaron para extraer información sobre competencias procedimentales/declarativas, condicionales, afectivas y socioculturales de la escritura, según el Modelo Metasociocognitivo de la Composición Escrita (Arroyo, 2009). La Tabla 3 reproduce parte de esa entrevista.

Esta entrevista se aplicó después de realizar la tarea de escribir un ensayo científico. Los estudiantes tuvieron una hora para su realización en aulas asignadas. El profesor estuvo en todo momento presente para solucionar cualquier duda que surgiese en el transcurso de la tarea.

Tabla 3

Entrevista Metasociocognitiva sobre Competencias Escritoras

\begin{tabular}{|c|c|}
\hline Items/operación escritoras & $\begin{array}{l}\text { Proceso/ } \\
\text { Competencia }\end{array}$ \\
\hline 19. Cuando escribes un texto piensas que ese texto ¿es útil para alguien? & Declarativo \\
\hline ¿Para quién? ¿Qué utilidad puede tener? Pon algún ejemplo & Teoría de la \\
\hline $\begin{array}{l}\text { 20. Cuando escribes un texto ¿piensas que el tema, la forma y el idioma en el } \\
\text { que se escribe está relacionado con la utilidad que tenga el texto? Pon algún } \\
\text { ejemplo }\end{array}$ & Tarea \\
\hline $\begin{array}{l}\text { 21. Cuando escribes un texto ¿tienes estrategias para lograr terminarlo en la } \\
\text { forma y con las ideas que tú querías? ¿Qué estrategias? Pon algún ejemplo }\end{array}$ & \\
\hline
\end{tabular}

Nota: Adaptado de Arroyo (2009).

Posteriormente esta entrevista fue sometida a la valoración por 12 jueces expertos. Mediante Escalas Likert (valores comprendidos entre "1", la valoración más negativa, y "4" la valoración más positiva). Las valoraciones de los jueces fueron sometidas al análisis $\mathrm{W}$ de Kendall, destacando el elevado acuerdo de los expertos respecto de los diferentes criterios (Arroyo \& Gutiérrez-Braojos, 2013).

\section{Procedimiento de recogida y análisis de datos}

Los estudiantes de la muestra fueron captados mediante la oferta de un seminario de escritura científica en la Facultad de Ciencias de la Educación de la Universidad de Granada. Unas de las tareas de este seminario fueron: a) la realización de un ensayo científico y b) la aplicación de una entrevista cognitiva individual para obtener información de sobre el conocimiento de competencias escritoras del estudiante, que se describen seguidamente.

El procedimiento aplicado para recoger los ensayos de los estudiantes fue una plantilla. En la aplicación de este instrumento, el profesor estuvo, en todo momento, presente para solucionar cualquier duda que surgiese en el transcurso de la tarea. Los estudiantes tuvieron dos horas para su realización en aulas asignadas. 
Para el análisis de los datos textuales se aplicó el Método de Análisis de Contenido siguiendo las siguientes fases:

Fase 1. Determinar las unidades a analizar. El universo de documentos estuvo compuesto por las plantillas que recogían los ensayos científicos escritos por los estudiantes y las entrevistas cognitivas sobre competencias escritoras. Las unidades de contexto estaban constituidas por la información registrada en cada entrevista, que ocupaba una media de tres páginas por cada sujeto, así como, los ensayos escritos que ocupaban una media de una página por sujeto. Por último, se establecieron como unidades de registro: la palabra, la frase o el párrafo; siempre que aportasen información completa sobre alguna competencia escritora o algún un elemento estructural del ensayo científico.

Fase 2. Clasificar la información textual. La clasificación de los datos contenidos en los documentos, se hizo asignando a cada unidad de registro un código de los sistemas de categorías, utilizados para ese fin. Por un lado, el Sistema de Categorías de Competencias Escritoras (véase Tabla 4) fue operativizado vía inductiva, es decir, las operaciones que se realizan en cada una de las competencias escritoras están definidas en base a una investigación previa en la que se aplicó, dicha entrevista, a 86 estudiantes (Arroyo, 2013).

Tabla 4

Sistema de Categorías de Competencias Escritoras

\begin{tabular}{llc}
\hline & Competencias Escritoras & Códigos \\
\hline 1 & Planificación de la escritura & $\mathrm{Sp}$ \\
2 & Transcripción de la escritura & $\mathrm{St}$ \\
3 & Revisión de la escritura & $\mathrm{Sr}$ \\
4 & Teoría de la Tarea escritora & Sta \\
5 & Teoría del Texto escrito & Stx \\
6 & Autorregulación de la escritura & Sarr \\
7 & Autocontrol de la escritura & Sac \\
8 & Motivación para la escritura & Sm \\
9 & Creatividad en la escritura & Sc \\
10 & Condicionamiento político en la escritura & Ss \\
11 & Condiciones comunitarias y/o profesionales en la escritura & Scp \\
12 & Construcción de la identidad en la escritura & Si \\
\hline
\end{tabular}

Nota: Adaptado de Arroyo (2013).

Posteriormente el Sistema de Categorías de Competencias Escritoras fue sometido a una validación por expertos, con el propósito de analizar el grado de acuerdo entre los jueces. Para ello se aplicó W de Kendall. Los resultados obtenidos en exhaustividad, exclusión mutua, pertinencia y productividad de cada competencia demuestra una claro acuerdo interjueces (Arroyo \& Gutiérrez-Braojos, 2013). 
El Sistema de Categorías de la Estructura del Ensayo Científico (ver Tabla 5) se elaboró vía deductiva en un grupo de discusión compuesto por miembros del Grupo de Investigación ED.INVEST (Hum 356), que luego fue validado empíricamente en esta investigación, revelándose como un potente instrumento para clasificar toda la información contenida en las unidades de contexto analizadas con el Programa Nvivo.

Esta investigación también ha permitido operativizar cada categoría tal y como aparece en la Tabla 5.

Tabla 5

Sistema de Categorías de Competencias Escritoras

\begin{tabular}{|c|c|c|c|}
\hline \multicolumn{4}{|c|}{ Sistema de Categorías de la Estructura del Ensayo Científico } \\
\hline Categorías & Subcategorías & Operación & Código \\
\hline \multirow[t]{4}{*}{ Introducción } & $\begin{array}{l}\text { 1. Presentación del } \\
\text { tema }\end{array}$ & Expone del tema del que trata el ensayo & Ipt \\
\hline & 2. Interés personal & $\begin{array}{l}\text { Explica por qué es importante este tema para el autor, en rel- } \\
\text { ación con su trayectoria formativa y su experiencia }\end{array}$ & $\operatorname{Irp}$ \\
\hline & $\begin{array}{l}\text { 3. Relevancia } \\
\text { social }\end{array}$ & $\begin{array}{l}\text { Explica por qué es importante ese tema para la sociedad, a qué } \\
\text { colectivos puede beneficiar y por qué }\end{array}$ & Irs \\
\hline & 4. Citas tema & $\begin{array}{l}\text { Aporta ideas o palabras textuales de expertos en el tema del en- } \\
\text { sayo, que apoyen las ideas expuestas }\end{array}$ & Iiv \\
\hline \multirow[t]{2}{*}{ Premisa } & $\begin{array}{l}\text { 5. Formulación de } \\
\text { la premisa }\end{array}$ & $\begin{array}{l}\text { Presenta de forma clara y concisa, en una afirmación, la idea que } \\
\text { se pretende argumentar }\end{array}$ & Pfp \\
\hline & $\begin{array}{l}\text { 6. Definición de } \\
\text { conceptos premisa }\end{array}$ & $\begin{array}{l}\text { Establece el significado exacto de los términos que se utilizan } \\
\text { en la premisa }\end{array}$ & Pdc \\
\hline \multirow[t]{6}{*}{ Argumentación } & 7. Razones a favor & $\begin{array}{l}\text { Construye argumentos que demuestran la veracidad de las rela- } \\
\text { ciones establecidas en la premisa }\end{array}$ & Arf \\
\hline & $\begin{array}{l}\text { 8. Razones en } \\
\text { contra }\end{array}$ & $\begin{array}{l}\text { Expone posibles argumentos que contradicen o muestren la in- } \\
\text { consistencia de las relaciones establecidas en la premisa. }\end{array}$ & Arc \\
\hline & 9. Refutaciones & $\begin{array}{l}\text { Refuerza la veracidad de la premisa con argumentos que expre- } \\
\text { sen la debilidad de las razones en contra de la premisa }\end{array}$ & Acr \\
\hline & 10. Citas nuevas & $\begin{array}{l}\text { Aporta ideas o palabras textuales de expertos en el tema del en- } \\
\text { sayo, que apoyen los argumentos expuestos }\end{array}$ & Act \\
\hline & $\begin{array}{l}\text { 11. Definición de } \\
\text { conceptos nuevos }\end{array}$ & $\begin{array}{l}\text { Establece el significado exacto de los nuevos términos que apa- } \\
\text { rezcan en la argumentación. }\end{array}$ & Adc \\
\hline & 12. Investigaciones & Aporta investigaciones que apoyan la argumentación & Aiv \\
\hline \multirow[t]{3}{*}{ Conclusión } & $\begin{array}{l}\text { 13. Síntesis de } \\
\text { razones }\end{array}$ & $\begin{array}{l}\text { Describe de forma sintética los argumentos expuestos más } \\
\text { destacados, que apoyan la premisa }\end{array}$ & Csr \\
\hline & $\begin{array}{l}\text { 14. Razón defini- } \\
\text { tiva }\end{array}$ & $\begin{array}{l}\text { Construye un argumento concluyente sobre la veracidad de las } \\
\text { relaciones establecidas en la premisa }\end{array}$ & Crd \\
\hline & $\begin{array}{l}\text { 15. Aplicación/ } \\
\text { Proyección }\end{array}$ & $\begin{array}{l}\text { Expresar las posibles implicaciones prácticas y las nuevas líneas } \\
\text { de conocimiento sobre el tema que se derivan de la premisa }\end{array}$ & Cap \\
\hline Bibliografía & $\begin{array}{l}\text { 16. Referencias } \\
\text { Bibliográficas }\end{array}$ & $\begin{array}{l}\text { Referencias exactas de las citas e investigaciones mencionadas } \\
\text { en el ensayo, siguiendo las normas de la APA }\end{array}$ & $\mathrm{Rbl}$ \\
\hline
\end{tabular}


Fase 3. Establecer reglas de recuento. Para la reducción de los datos textuales clasificados y codificados, se aplicó el recuento de frecuencias directas de cada código. De este modo se pudo obtener tablas tanto, de las frecuencias directas de las competencias escritoras expresadas por los sujetos, como de los elementos estructurales identificados en los productos escritos. También se calcularon los tantos por cientos en relación a la frecuencia total de categorías expresadas.

Para el análisis de los datos numéricos, obtenidos a partir del recuento de frecuencias, se aplicó estadísticos, tales como: test de normalidad Shapiro-Wilks, el coeficiente de correlación no paramétrico de Spearman y el estadístico de contraste U de MannWhitney.

En definitiva, en esta investigación se aplica técnicas de recogidas de datos cualitativas, reduciendo los datos en un modo cuantitativo para después hacer interpretaciones integradas en función de los objetivos, como se verá en el apartado de resultados.

\section{Resultados}

Seguidamente se presentarán los datos obtenidos, tras su análisis, de forma reducida en tablas y gráficos, a fin de poder realizar la interpretación de los mismos en base a los objetivos plateados.

\section{Contenido de los diferentes elementos estructurales expresados en los ensayos científicos producidos por estudiantes universitarios}

En este apartado se interpretan los elementos estructurales del ensayo científico, en función de su frecuencia proporcional y mostrando ejemplos del contenido expresado en cada elemento. Para ello se extraerán citas de los textos analizados con el programa Nvivo. Cada cita se identifica con un código del tipo: (T01); donde T01, hace referencia al número que se asignó al texto del cual se extrae la cita.

Si se observa la Tabla 6 y la Figura 1, los elementos estructurales que se expresan con más frecuencia en los textos analizados son, en primer lugar, las razones a favor con una frecuencia del 21,30\%. Sin embargo, analizado el contenido de este elemento, se descubre que, en realidad, se tratan de afirmaciones generales, sin progresión y diferenciación de ideas. Más que formular razones que demuestran una premisa previamente bien formulada, directamente se formulan afirmaciones del tipo: «La cultura de los pobres es estigmatizada por sectores de la sociedad como inferior, precaria, atrasada" (T01); con lo que el lector debe de suponer que el ensayista trata de argumentar una premisa del tipo "La cultura desvalorizada crea baja autoestima".

En segundo lugar (ver Figura 1) aparece el elemento estructural: la aplicación o proyección de las conclusiones; con una frecuencia del 17\%. Se descubre en el contenido de este elemento, recomendaciones de carácter general y estereotipadas políticamente que, sugieren un proceso sin coherencia ni progresión argumental. Un ejemplo de este tipo de proyección se muestra en el siguiente ejemplo: "El sistema educativo debe hacer suyas las necesidades de comunicación y de intercambio surgidas en una sociedad más abierta como la actual, donde las diferentes culturas se mezclan" (T23). 
Tabla 6

Frecuencias de los elementos estructurales

\begin{tabular}{llll}
\hline Elementos Estructurales & Código & Frecuencia & $\%$ \\
\hline 1. Presentación del tema & Ipt & 22 & 4.9 \\
2. Interés personal & Irp & 20 & 4.48 \\
3. Relevancia social & Irs & 32 & 11.21 \\
4. Citas tema & Iiv & 2 & 0.44 \\
5. Formulación de la premisa & Pfp & 53 & 11.88 \\
6. Definición de conceptos premisa & Pdc & 25 & 5.60 \\
7. Razones a favor & Arf & 95 & 21.30 \\
8. Razones en contra & Arc & 32 & 7.17 \\
9. Refutaciones & Acr & 17 & 3.81 \\
10. Citas nuevas & Act & 6 & 1.37 \\
11. Definición conceptos nuevos & Adc & 3 & 0.67 \\
12. Investigaciones & Aiv & 19 & 4.69 \\
13. Síntesis de razones & Csr & 3 & 0.67 \\
14. Razón definitiva & Crd & 18 & 4.03 \\
15. Aplicación/Proyección & Cap & 80 & 17.93 \\
16. Referencias bibliográficas & Rbl & 1 & 0.22 \\
\hline Total & & 446 & 100 \\
\hline
\end{tabular}

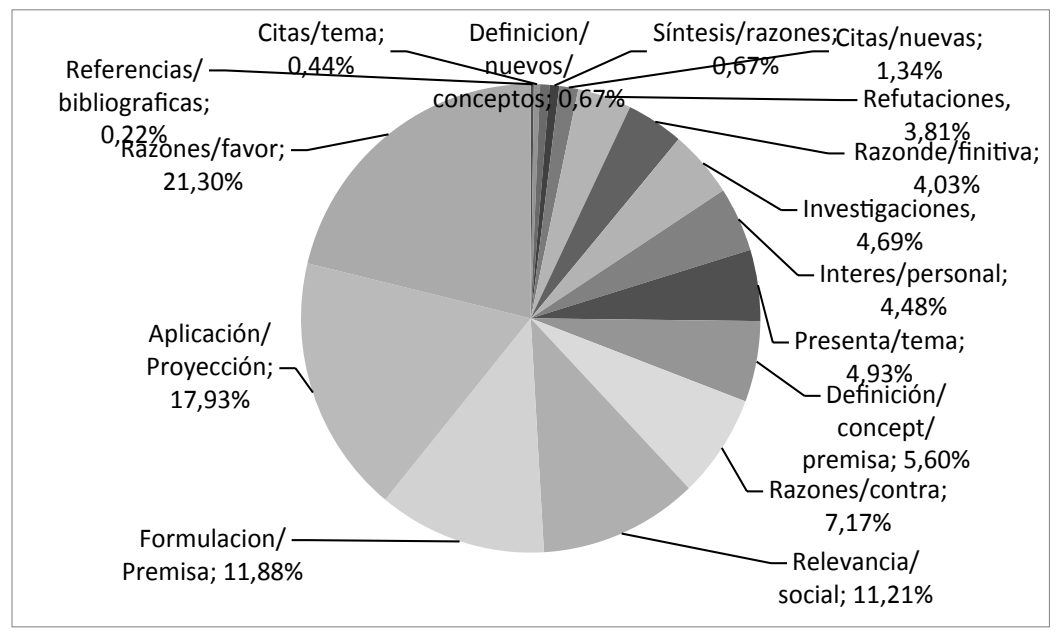

Figura 1. Frecuencias directas y porcentajes de los elementos estructurales del ensayo científico, identificados en los textos de la muestra de estudiantes universitarios.

En el tercer lugar de frecuencias se encuentran dos elementos estructurales: A) la formulación de la premisa con una frecuencia del 11,88\% y, B) la relevancia social con una frecuencia del 11,21\%, (ver Figura 1). 
A. En relación a la formulación de la premisa, su contenido destaca que, si bien, un alto porcentaje de textos la incluyen; se formula en forma de opinión sin la pretensión, ni el convencimiento formal de ser demostrada científicamente. Un ejemplo, es la premisa del tipo: "En mi opinión, creo que los colegios que siguen un modelo de enseñanza multilingüe bajan el nivel de exigencia en otras materias" (T32).

B. En relación a la relevancia social, el contenido de este elemento estructural destaca la importancia que tiene el tema que se están tratando para el sujeto que escribe. Se puede afirmar que es en este elemento donde los ensayistas universitarios se muestra más conscientes, como se constata en los siguientes ejemplos: "En la actualidad y, quizás más que nunca, ante los nuevos cambios que se están produciendo a nivel mundial...se hace cada vez más necesario el aprendizaje de distintas lenguas" (T42). "A la enseñanza multilingüe en la actualidad se le concede mucha importancia, tal es esta que presenta un día oficial el 23 de septiembre" (T2).

En cuarto lugar, las razones en contra, se presentan con una frecuencia del 7,17\% (ver Figura 1). Analizado el contenido de las razones en contra, se concluye que los estudiante expresan contra-argumentos a las razones que sostienen, aunque expresando opiniones no contrastadas, como por ejemplo: "la mayoría de las personas multilingües tienen faltas de expresión oral y/o escrita, ya que se encuentran influenciadas por alguna lengua, y carecen de competencia lingüística en otra/s lengua/s" (T43). Sin embargo, algunas razones en contra son muy acertadas, mostrándose un importante nivel de reflexión, como se comprueba en los ejemplos siguientes: "El otro extremo podría ser la consideración de que todo da igual, de que todas las culturas son iguales... en una especie de homogeneización... la que las modas y las fuerzas más organizadas puedan, a través de medios de comunicación y publicidad, soslayar determinados valores y actitudes" (T03).

Por otra parte, la refutación se presenta con una frecuencia del 3,81\%; y suelen ser, más estimaciones que demostraciones. Para ilustrar esto, baste este ejemplo: "los hechos han demostrado que es un grave error y que se debe caminar hacia una sociedad plural $y$ diversa" (T04). Aunque no faltan ejemplo en el que se intentan formular auténticas demostraciones que refuten contra-argumentos: "Pero solo hay que fijarse en el éxito de los programas seguidos en Canadá, gracias al compromiso con el multilingüismo, metodología, preparación del equipo docente..." (T09).

En el quinto lugar se sitúa la definición de conceptos dentro de la premisa con una frecuencia del 5,60\%, (ver Figura 1). En los textos analizados se definen los siguientes conceptos: cultura, colaboración, cooperación, diversidad cultural, progreso, multilingüismo, enseñanza multilingüe, plurilingüismo, lengua extranjera, inmersión lingüística, colegio bilingüe; en concordancia temática con las premisas que se formulan, y en general estas definiciones suelen mostrar aproximación intuitiva, como en el ejemplo que sigue: "La enseñanza multilingüe pretende ayudar a la comprensión y a facilitar un acceso mejorado a la educación a personas de diferente índole cultural " (T11. En otro casos, en la definición se reproducen estereotipos sociales del tipo: "una enseñanza multilingüe, es decir, que los alumnos tengan el conocimiento de lenguas que se mantienen en compartimentos separados" (T31).

En sexto lugar, se identifican una serie de elementos estructurales, con una frecuencia que oscila entre en el 4,93\% y el 4,03\%, (ver Figura 1), por lo tanto, con poca significación, porque son expresados en menos de la mitad de los textos, (Ver Figura 1). Estos 
elementos son: a) la presentación del tema, (en 16 textos); b) el interés personal, (en 18 textos); c) cita de investigaciones, (en 13 textos); d) razón definitiva, (en 18 textos).

Además señalar que las citas de expertos sobre el tema, las citas nuevas y la definición de nuevos conceptos en la argumentación, la síntesis de razones y las referencias bibliográficas, presentan una frecuencia entre 1,34\% y el 0,22\%, (ver Figura 1); lo que supone que parecen entre 6 y 2 textos, del total de la muestra analizada.

Para completar el conocimiento en profundidad sobre la escritura del ensayo científico, interesa conocer, no solo cómo se estructura el contenido de los textos argumentativos, sino también, como correlaciona con las competencias metasociocognitivas de la escritura que el autor del ensayo muestra. De esto se ocupa el apartado siguiente.

\section{Correlación entre los contenidos estructurales de los ensayos científicos y la expre- sión de competencias escritoras}

En la Tabla 7 se presentan las competencias lingüísticas, cognitivas, afectivas y sociales, expresadas por los estudiantes de la muestra analizada.

Tabla 7

Tabla de porcentajes de las competencias escritoras

\begin{tabular}{cccccccccccccc}
\hline Comp & Sp & St & Sr & Sta & Stx & Sarr & Sac & Sm & Sc & Ss & Scp & Si & ST \\
\hline$\%$ & 22.12 & 12.50 & 8.65 & 6.12 & 5.18 & 7.09 & 3.6 & 10.74 & 2.14 & 3.24 & 10.12 & 8.50 & $\mathbf{1 0 0}$ \\
\hline
\end{tabular}

Nota:

Sp: Sumatorio de competencias de planificación en la escritura

St: Sumatorio de competencias de transcripción en la escritura

Sr: Sumatorio de competencias de revisión en la escritura

Sta: Sumatorio de competencias de teoría de la tarea en la escritura

Stx: Sumatorio de teoría del texto en la escritura

Sarr: Sumatorio de competencias de autorregulación en la escritura

Sac: Sumatorio de competencias de autocontrol en la escritura

Sm: Sumatorio de competencias de motivación en la escritura

Sc: Sumatorio de competencias de creatividad en la escritura

Ss: Sumatorio de competencias sociales en la escritura

Scp: Sumatorio de competencias comunitarias-profesionales en la escritura

Si: Sumatorio de competencias de identidad en la escritura

ST: Suma total de frecuencias expresadas en competencias escritoras en $\%$

Seguidamente se establecerá como correlaciona la estructuración del ensayo científico con las competencias escritoras expresadas por los estudiantes.

En la Tabla 8 se presentan los estadísticos descriptivos para las variables competencias y elementos estructurales, así como los resultados del test de normalidad ShapiroWilks. Debido a que ambas variables no fueron normales, se utilizó el coeficiente de correlación no paramétrico de Spearman, que tiene el mismo significado que el coeficiente de correlación de Pearson y se calcula utilizando el rango de las observaciones para evaluar la asociación entre ambas variables. 
Tabla 8

Estadísticos descriptivos para variables de estudio $(N=43)$. Se indica además los resultados del test de normalidad de Shapiro-Wilks (SW)

\begin{tabular}{llllll}
\hline & Mínimo & Máximo & Media & DT & SW \\
\hline Elementos estructurales & 4 & 21 & 10.37 & 3.879 & $0.945(\mathrm{p}=0.040)$ \\
Competencias escritoras & 19 & 258 & 81.84 & 36.050 & $0.832(\mathrm{p}<0.001)$ \\
\hline
\end{tabular}

Aplicando el coeficiente de correlación no paramétrico de Spearman entre las `competencias escritoras' y los 'elementos estructurales' se obtuvo que no fue significativa $(\mathrm{rs}=0.072, \mathrm{p}=0.646)$. La relación entre ambas puede observarse la Figura 2,

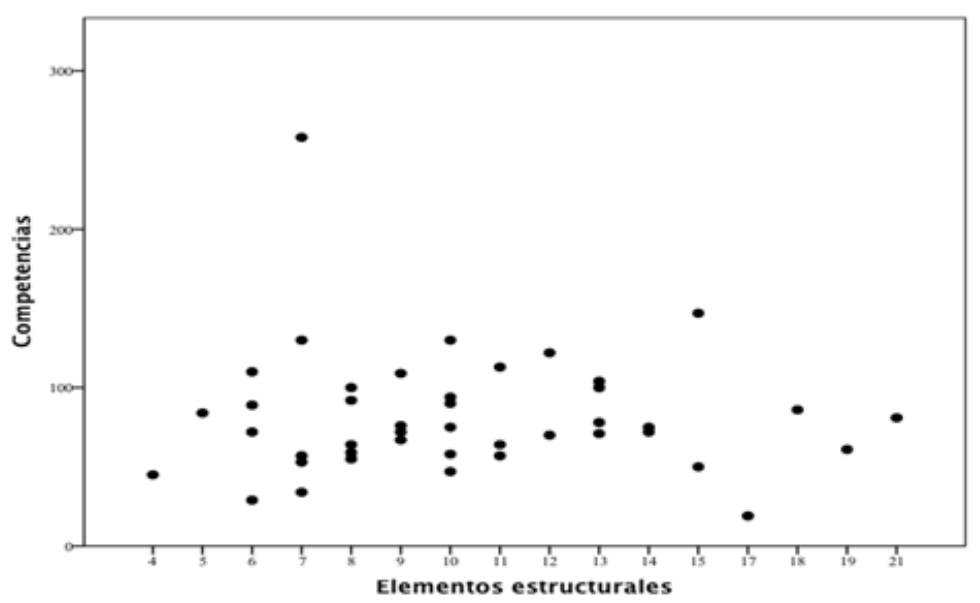

Figura 2. Gráfico de dispersión entre competencias escritoras y elementos estructurales

donde claramente se aprecia la dispersión. Por lo tanto, se cumple la hipótesis H1: el conocimiento de competencias escritoras expresado por una muestra de estudiantes universitarios no correlaciona con los elementos estructurales de los ensayos científicos producidos por ellos mismos. Esto significa que aquellos alumnos que expresaron mayores frecuencias en competencias escritoras no fueron mayores también las frecuencias en elementos estructurales y viceversa.

En el apartado siguiente se comprobará en qué medida afecta a la estructuración del ensayo científico en el paso por diferentes cursos universitarios.

\section{Las diferencias en elementos estructurales por niveles}

En la Tabla 9 se indican los estadísticos descriptivos de la variable 'elementos estructurales', para los dos niveles académicos estudiados. En ambos grupos la variable no siguió una distribución normal y por ende se utilizó un test no paramétrico para comparar ambos grupos. 
Tabla 9

Estadísticos descriptivos de la variable 'elementos estructurales' según el nivel

\begin{tabular}{llllll}
\hline Curso & $\mathrm{N}$ & Mínimo & Máximo & Media & Desv. típ. \\
\hline $1^{\mathrm{o}}$ curso & 26 & 5 & 21 & 10.62 & 3.940 \\
$3^{\mathrm{o}} \mathrm{y} 4^{\mathrm{o}}$ curso & 17 & 4 & 18 & 10.00 & 3.873 \\
\hline
\end{tabular}

El estadístico de contraste U de Mann-Whitney indicó que no existen diferencias significativas en los 'elementos estructurales' entre ambos grupos ( $\mathrm{U}=198.500, \mathrm{p}=0.575)$. En consecuencia queda confirmada la Hipótesis 2: Los elementos estructurales de los ensayos científicos escritos por estudiantes universitarios de cursos diferentes no presentan diferencias significativas. Es decir los estudiantes de cursos universitarios superiores no incluyen más elementos estructurales en sus ensayos.

\section{Discusión y conclusiones}

En este apartado se caracterizará la escritura del ensayo científico de los sujetos de la muestra, atendiendo a la variable 'estructuración del texto' y sus posibles diferencias según el nivel universitario de los sujetos. Además, se identificarán la correlación con las competencias escritoras expresadas

En primer lugar, con respecto a los diferentes elementos estructurales, expresados en los textos, se puede afirmar que los ensayos científicos de los estudiantes analizados se centran en desarrollar razones a favor, que apoyen unas premisas no explicitadas de una forma clara e inequívoca para ser demostradas. Estos datos se corresponden con un estudio sobre los ensayos realizado con una muestra de 32 estudiantes universitarios donde se concluye la ausencia de una estructura clara (Greasley \& Cassidy, 2010).

Otro elemento que se destaca en la escritura de ensayos universitarios, es la expresión de una proyección o aplicación de carácter educativo del tema planteado; aceptada por la tendencia sociocultural del momento. Es decir en cuanto a contendidos interculturales solo se expresan ideas estereotipadas sobre las relaciones entre variables sociales, económicas, políticas y culturales. Además, se pueden identificar premisas, contra-argumento, refutaciones y definición de conceptos, frecuentemente carentes de rigor, sin embargo se insiste en la relevancia social del tema que tratan.

En segundo lugar se comprueba que aquellos estudiantes que expresan más elementos estructurales en sus ensayos científicos, no presentan mayor frecuencia de competencias escritoras y viceversa, por lo que en esta muestra, la estructuración del texto es independiente del conocimiento que el sujeto muestra sobre sus competencias escritoras.

Por último también se comprueba que el paso por diferentes cursos en la enseñanza universitaria no afecta al expresión de los elementos estructurales del ensayo científico, 
corroborando que la práctica regular de la escritura, en estos niveles, no es suficiente para producir textos que reúnan los criterios de calidad exigidos (Walker, Golde, Jones, Bueschel \& Huntchingsl, 2008).

Además de lo expuesto se identifican importantes necesidades formativas del estudiante universitario en la construcción de ensayos científicos como son: a) la carencia de una presentación del tema a tratar, describiendo el interés personal por el mismo; b) la escasa presencia de citas de expertos e investigaciones; c) la ausencia de una diferenciación y progresión ordenada de las ideas que conduzca a síntesis finales y razones definitivas; y d) la inexistencia de referencias bibliográficas.

Un estudio realizado por Sydney (2014) demuestra, con una muestra de 22 ensayos, que los estudiantes universitarios sin una experiencia previa formativa en la construcción de textos argumentativos científicos, reproducen las estructuras de los textos leído en el contexto académico, por lo tanto, las necesidades formativas de los estudiantes investigación está sugiriendo que, además de no haber pasado por un proceso de formación en la estructuración del ensayo científicos, los textos, que habitualmente leen en las materias universitarios, no ofrecen modelos suficientemente estructurados.

Estas necesidades formativas expresas, unidas a que no existe una asociación entre las competencias escritoras que describe el estudiante, con su habilidad para estructurar el texto, por un lado; y a que esta estructuración no se ve afectada por la experiencia universitaria; está indicando, claramente, la necesidad de un intervención didáctica en este nivel formativo que incida en la enseñanza de competencias escritoras para la adecuada estructuración del ensayo científico.

Sin duda, esta investigación presenta la limitación común en investigaciones educativas en profundidad y es la imposibilidad de generalizar los resultados, por centrarse en muestras pequeñas y no aleatorias. Sin embargo, si se está constatando un campo de necesidad en la formación universitaria que puede ser transferible en su intervención didáctica. A este fin, subvencionado por la Universidad de Granada y en el marco del Grupo de Investigación EDINVEST (HUM356) de la Junta de Andalucía, se está diseñando un OCW para la enseñanza del ensayo científico multilingüe (español, inglés, alemán, italiano y catalán), que atienda las necesidad detectadas y pueda ser aplicado y evaluado en otras universidades nacionales e internacionales.

\section{Referencias}

Arroyo, R. (2009). Desarrollo Metacognitivo y Sociocultural de la Composición Escrita. Interculturalidad y Tecnologías en la enseñanza de la escritura multilingüe. Granada: Nativola.

Arroyo, R. (2013). Descripción de procesos en la composición escrita de estudiantes universitarios para un desarrollo multilingüe y tecnológico. Revista de Investigación Educativa, 31(1), 167-184.

Arroyo, R. \& Gutiérrez-Braojos, C. (2013). Validación de una entrevista metasociocognitiva y un sistema de categorías, para la investigación multilingüe de procesos escritores. Educación y Diversidad. Revista Interuniversitaria de Investigación en Discapacidad e Interculturalidad, 7(2), 67-86.

Bazerman, C. (Ed.). (2008). Handbook of research on writing. Mahwah, NJ: Erlbaum. 
Beaufort, A. \& Iñesta, A. (2014). Author profiles: Awareness, competence, and skills. En E. M. Jakobs \& D. Perrin. (Eds.), Handbook of Writing and Text Production (pp. 141-158). Berlín/Boston: Walter de Gruyter.

Cernadas, F., Santos, M. \& Lorenzo, M. (2013). Los profesores ante la educación intercultural: el desafío de la formación sobre el terreno. Revista de Investigación Educativa, 31(2), 555-570.

Cleaveland, M. C. \& Larkins, E. R. (2004). Web-based practice and feeback improve tax students' written communication skills. Journal of Accounting Education, 22, 211-228. doi:101016/j.jaccedu.2004.08.001.

De La Paz, S. \& McCutchen, D. (2010). Learning to Write. In R. E. Mayer \& P. A. Alexander (Eds). Handbook of Research on Learnig and Instruction, 32-54. Routledge. New York.

Dysthe, O. (2007). How a Reform Affects Writing in Higher Education. Studies in Higher Education, 32(2), 237-252.

Kieft, M., Rijlaarsdam, G. \& Van Den Bergh, H. (2006). Writing as a learning tool: Testing the role of students' writing strategies. European Journal of Psychology of Education, 21(1), 17-34.

Gavin, B. \& Marshall, J. (2012). The impact of training students how to write introductions for academic essays: an exploratory, longitudinal study. Assessment $\mathcal{E}$ Evaluation in Higher Education, 37(6), 653-670. doi: 10.1080/02602938.2011.563277

Graham, S., \& Harris, K. R. (2005). Writing better: Teaching writing processes and selfregulation to students with learning problems. Baltimore: Brookes.

Greasley, P. \& Cassidy, A. (2010). When it comes round to making assignments: How to impress and how to 'distress' lecturers. Assessment \& Evaluation in Higer Education, 35(2), 173-89.

Mayorga, M.J. \& Ruiz Baeza, V.M. (2002). Muestreos utilizados en la investigación educativa en España. Revista Electrónica de Investigación y Evaluación Educativa, $8(2), 1-8$.

Myhill, D. \& Fisher, R. (2010). Writing development: cognitive, sociocultural, linguistc perspectives. Journal of Research in Reading, 33(1), 1-3.

Nussbaum, E.M. \& Kardash, C. M. (2005). The effects of goal instructions and text on the generation of counterarguments during writing. Journal of Educational Psychology, 97( 2), 157-16.

Salvador, F. (2005). Procesos cognitivos en la expresión escrita: Modelos teóricos e investigación empírica. En F. Salvador, (Ed.), La expresión escrita de alumnos con necesidades educativas especiales. Procesos cognitivos (pp. 15-44). Archidona: Aljibe.

Salvador, F. (2008). Competencia escritora del alumno universitario: El texto argumentativo (ensayo). V Congreso Iberoamericano de Docencia Universitaria AIDU e ICE, Valencia (pp. 907-910). Valencia.

Sydney, R. (2014). Academic essay writing as imitative problem solving: examples from distance learning. Assessment E Evaluation in Higher Education, 39(3), 263-274. doi: 10.1080/02602938.2013.822846

Takao, A. Y. \& Kelly, G.J. (2003). Assessment of Evidence in University Students' Scientific Writing. Science E Education, 12, 341-363. 
Tashakkori, A. \& Teddlie, C. (2003). Handbook of mixed methods in social and behavioral research. Thousand Oaks, CA: Sage.

Venables, A. \& Summit, R. (2003). Enhancing Scientific Essay Writing Using Peer Assessment. Innovations in EducationandTeachingInternational, 40(3), 281-290.

Walker, G. E., Golde, C. M., Jones, L., Bueschel, A.C., \& Huntchings, P. (2008). The formation of scholars: Rethinking doctoral education for the twenty-first century. San Francisco, CA: Jossy-Bass.

Fecha de recepción: 23-06-2015

Fecha de revisión: 25-06-2015

Fecha de aceptación: 13-01-2016 
